\title{
Comment on "COVID-19 severe acute respiratory syndrome coronavirus 2 (SARS-CoV-2) infection in children and adolescents: a systematic review of critically unwell children and the association with underlying comorbidities"
}

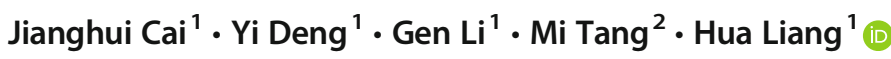 \\ Received: 23 February 2021 / Revised: 23 February 2021 / Accepted: 16 March 2021 / Published online: 25 March 2021 \\ (C) The Author(s), under exclusive licence to Springer-Verlag GmbH Germany, part of Springer Nature 2021
}

Dear Editor,

We read with great interest the review by Williams and colleagues (Volume 180, issue 3, March 2021) [1]. The authors identified and described which underlying comorbidities may be associated with SARS-CoV-2 disease and death. The authors should be applauded for the critical emerging evidence they provide. However, we raise several concerns regarding this systematic review.

First, the statements of "The study protocol was in keeping with Preferred Reporting Items for Systematic Reviews and Meta-analyses (PRISMA) guidelines" in the Abstract and Study selection should read "The study protocol was in keeping with Preferred Reporting Items for Systematic Reviews and Meta-analyses Protocols (PRISMA-P) guidelines" [2] or "The study was in keeping with Preferred Reporting Items for Systematic Reviews and Meta-analyses (PRISMA) guidelines" [3]. Although a minor issue, this should be clarified.

Second, the authors should clearly state what laboratoryconfirmed SARS-CoV-2 infection is. Did a laboratoryconfirmed case mean a positive result to reverse transcriptasepolymerase chain reaction (RT-PCR) nasopharyngeal swab and/or antibody testing SARS-CoV-2? The authors should

Communicated by Peter de Winter

Hua Liang

lianghuacd@126.com

1 Department of Pharmacy, Chengdu Women's and Children's Central Hospital, School of Medicine, University of Electronic Science and Technology of China, No.1314 Riyue Avenue, Qingyang District, Chengdu City 610041, China

2 Office of Good Clinical Practice, Chengdu Women's and Children's Central Hospital, School of Medicine, University of Electronic Science and Technology of China, Chengdu 610041, China make it clear and adopt a uniform case definition throughout the manuscript.

Third, only MEDLINE (PubMed) databases were used for potential articles, which may miss important or relevant studies. What's more, the authors should present a full electronic search strategy for at least one database as online supplemental data, including any limits used, such that it could be repeated [3].

Four, we are very surprised to find that the authors included a study (reference 9) that was pooled from a national registry from 50 states, the District of Columbia, and four US territories [4]. Thus, the authors didn't exclude studies suspected of including duplicate reporting. We suggest that the authors should review the hospital and periods of recruitment. If they overlapped, only the study with the biggest data was included to minimize the possibility of double counting.

Five, the authors didn't assess the risk of bias in individual studies according to the PRISMA recommendations, which are mandatory for any systematic review.

Authors' contributions All authors contributed equally to this work. All authors read and approved the final manuscript.

\section{Declarations}

Conflict of interest The authors declare no competing interests.

\section{References}

1. Williams N, Radia T, Harman K, Agrawal P, Cook J, Gupta A (2021) COVID-19 Severe acute respiratory syndrome coronavirus 2 (SARS-CoV-2) infection in children and adolescents: a systematic review of critically unwell children and the association with underlying comorbidities. Eur J Pediatr 180:689-697. https://doi.org/10. 1007/s00431-020-03801-6 
2. Moher D, Shamseer L, Clarke M, Ghersi D, Liberati A, Petticrew M, Shekelle P, Stewart LA (2015) Preferred reporting items for systematic review and meta-analysis protocols (PRISMA-P) 2015 statement. Syst Rev 4(1):1. https://doi.org/10.1186/2046-4053-4-1

3. Moher D, Liberati A, Tetzlaff J, Altman DG (2009) Preferred reporting items for systematic reviews and meta-analyses: the PRISMA statement. BMJ 339:b2535. https://doi.org/10.1136/bmj. b2535
4. CDC COVID-19 Response Team (2020) Coronavirus disease 2019 in children - United States, February 12-April 2, 2020. MMWR Morb Mortal Wkly Rep 69(14):422-426. https://doi.org/10.15585/ mmwr.mm6914e4

Publisher's note Springer Nature remains neutral with regard to jurisdictional claims in published maps and institutional affiliations. 